\title{
Identification of IgE and IgG1 specific antigens in Echinococcus granulosus cyst fluid
}

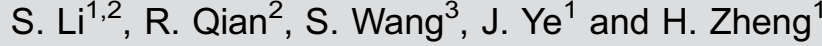 \\ ${ }^{1}$ Department of Anesthesiology, First Affiliated Hospital of Xinjiang Medical University, Urumqi, China \\ ${ }^{2}$ Department of Anesthesiology, General Hospital, Xinjiang Command PLA, Urumqi, China \\ ${ }^{3}$ Department of Immunology, Basic Medical College of Xinjiang Medical University, Urumqi, China
}

\begin{abstract}
Cystic echinococcosis (CE) is an anthropozoonotic disease with worldwide distribution and is caused by the cestode Echinococcus granulosus. Anaphylactic shock induced by CE rupture is a serious complication especially in patients with hydatid infections, as the resulting leakage of fluid contains highly toxic endogenous antigen. We aimed to isolate and identify the antigens of specific lgE and lgG1 (slgE and slgG1) in E. granulosus cyst fluid (EgCF). Crude antigen for EgCF was prepared from $E$. granulosus-infected sheep liver. Antigens were separated and identified by one-dimensional sodium dodecyl sulfatepolyacrylamide gel electrophoresis (1D SDS-PAGE), two-dimensional gel electrophoresis (2-DE), and immunoblotting. Results of 1D SDS-PAGE and immunoblotting showed that $40.5 \mathrm{kDa}$ protein was the major antigen of slgE, and $35.5 \mathrm{kDa}$ protein was the major antigen of slgG1 in EgCF. Results of 2-DE and immunoblotting showed that main antigens of slgE in EgCF were four proteins with pl values ranging from 6.5 to 9.0 and a molecular weight of $40.5 \mathrm{kDa}$. Main antigens of slgG1 in EgCF were five proteins with pl values ranging from 6.5 to 9.0 and a molecular weight of $35.5 \mathrm{kDa}$. The antigens identified for slgE and slgG1 can provide critical insights into cellular and molecular mechanisms underlying anaphylactic shock induced by $\mathrm{CE}$.
\end{abstract}

Key words: Cystic echinococcosis; Anaphylactic shock; Specific antigens; Specific antibody

\section{Introduction}

Cystic echinococcosis (CE) is a worldwide disease caused by the cestode Echinococcus granulosus. The prevalence of this disease in epidemic areas is estimated to be $1-7 \%(1)$, and the incidence rate is as high as $9 \%$ in some localities in Xinjiang, China (2). The overt or unapparent rupture of the parasitic cyst can cause anaphylactic shock, becoming a serious problem (3-5). Patients that develop type I hypersensitivity followed by echinococcosis-induced anaphylactic shock have specific clinical manifestations and immunological characteristics, and usually have poor responses to treatment and poor prognosis (6)

As the antigen components of the $E$. granulosus cyst fluid (EgCF) are complex, researchers have been unable to identify the allergen responsible for the anaphylactic shock; hence, it is difficult to treat the complications effectively. Experiments on animals found that in addition to immunoglobulin $E$ ( $\lg E)$, IgG1 antibody is also involved in type I hypersensitivity $(7,8)$. IgE-mast cells-histamine pathway has long been associated with anaphylaxis, but an alternative pathway mediated by IgG1 has been suggested to be more important in the elicitation of anaphylaxis (9). Early research suggests that, besides specific $\lg \mathrm{E}$ (slgE), anaphylactic shock and even death induced by $\mathrm{CE}$ are largely mediated by slgG, especially slgG1 subclass $(10,11)$. However, slgE and slgG1 antigens produced in EgCF are still not clearly known. Determining the levels of slgE and slgG1 in EgCF can be of great significance for assessing the risk of anaphylactic shock, and will certainly provide critical insights into cellular and molecular mechanisms underlying anaphylactic shock induced by CE.

In this study, we conducted experiments to identify the main antigens of slgE and slgG1 in EgCF, and to better understand cellular and molecular mechanisms underlying anaphylactic shock induced by CE

\section{Material and Methods}

\section{Patients and disease controls}

The study protocol was approved by the Ethics Committee of the First Affiliated Hospital of Xinjiang Medical University. Patients were mainly from the Altay, Ili Kazakh, Aksu, and Tacheng epidemic areas, and were diagnosed with echinococcosis by surgery at the institution. All patients and healthy volunteers provided written informed consent. In total, 20 patients (T1-T20) admitted for surgery and 10

Correspondence: H. Zheng: <xjzhenghong@aliyun.com> 
healthy volunteers (C1-C10) who served as disease controls recruited outside the epidemic area in the Urumqi General Hospital, Lanzhou Command, PLA, China, were enrolled into this study. The diagnosis of anaphylactic shock and classification standard for cysts were the same as used in previous studies $(6,11)$. Serum obtained from patients and healthy volunteers was repackaged and stored at $-80^{\circ} \mathrm{C}$ until further use.

\section{Inclusion and exclusion criteria}

Inclusion criteria. Cystic echinococcosis surgical patients who provided written informed consent.

Exclusion criteria. Patients who had other infectious diseases at the same time (such as bacterial liver abscess, or hepatitis); and patients who had underlying immune system disease.

Healthy volunteer exclusion criteria. Patients with infectious diseases (all kinds of hepatitis); with accompanied immune system disease; and with respiratory infections.

\section{Preparation of crude antigen of EgCF}

EgCF was obtained from infected sheep liver from the local slaughterhouses in Urumqi, China. Freshly isolated EgCF was stored in sterile containers and precipitated for $30 \mathrm{~min}$ to remove protoscoleces of $E$. granulosus and then centrifuged $(15,000 \mathrm{~g}$ for $30 \mathrm{~min}$ at room temperature). The supernatant used was $3 \mathrm{kDa}$, obtained by centrifugal ultrafiltration $(12,000 \mathrm{~g}$ for $20 \mathrm{~min}$ at room temperature) to obtain the crude antigen of EgCF. The quantity of crude antigen protein concentration used was $12.5 \mathrm{mg} / \mathrm{mL}$, which was repackaged and stored at $-80^{\circ} \mathrm{C}$ until use.

\section{Screening of serum-positive slgE and slgG1 through ELISA}

The ELISA experiment followed the method adopted by Liu et al. (12). Ninety-six well plates were coated with $120 \mu \mathrm{L}$ of $10 \mu \mathrm{g} / \mathrm{mL}$ crude antigen of EgCF. Determination of slgE concentration was done with patient sera (undiluted) with horseradish peroxidase (HRP)-labeled mouse anti-human IgE antibody added (Southern Biotech, USA; Lot No.: J681-RB83L 1:1000 dilution). Determination of concentration of slgG1 was done with patient sera (1:10 dilution), with HRP-labeled mouse anti-human lgG1 antibody (SouthernBiotech; Lot No.: L471-NB842 1:1000 dilution) added.

Serum from healthy volunteers was used as control. Absorbance was read at $450 \mathrm{~nm}$ on a microplate reader (xMark $^{\mathrm{TM}}$ Microplate Spectrophotometer; Bio-Rad, USA). All ELISA experiments were performed in duplicate, and the data obtained are reported as means \pm SD. Mean absorbance + three standard deviations from controls were used to establish a cutoff value. Patient values greater than the cutoff value were considered to be anti-EgCF slgE or slgG1 positive, and serum-positive specimens were used for further immunoblotting experiments.

\section{One-dimensional SDS-PAGE and $\lg E$ and $\lg G 1$ immunoblotting}

The basic experimental method used was described by Zheng et al. (10). Standard molecular protein markers were provided by Bio-Rad (range 10-250 kDa, Lot No. 161-0374) and Thermo Company (range 10-170 kDa, Lot No. 00102717, USA).

Electrophoresis was performed using a Mini-Protein 3 Cell (Bio-Rad), and the separated proteins were electrotransferred from gel to a nitrocellulose membrane (HybondC Extra RPN303E; Amersham Biosciences, Sweden) using Trans-Blot Electrophoretic Transfer Cell (Bio-Rad). The concentrated EgCF proteins were separated using 10\% SDSPAGE.

IgE immunoblotting. Patient sera (1:5 dilution) was added with HRP-labeled mouse anti-human IgE antibody (1:3000 dilution). Blots were developed using SuperSignal West Femto Maximum Sensitivity Substrate (Lot no: 34095 Thermo USA), and luminescence was detected on an X-ray film.

IgG1 immunoblotting. Patient sera (1:20 dilution) was added with HRP-labeled mouse anti-human IgG1 antibody (1:3000 dilution). Blots were visualized after staining with diaminobenzidine (DAB).

The results of immunoblotting were analyzed using Molecular Imager ${ }^{\circledR}$ Gel Doc ${ }^{\mathrm{TM}}$ XR + Imaging System (BioRad) with Image Lab 4.0.1 software.

\section{2-DE and immunoblotting}

The basic experimental method used was described by Liu et al. (12). To perform two-dimensional gel electrophoresis (2-DE) experiments combined with immunoblotting, $200 \mu \mathrm{g}$ of crude antigen of EgCF was diluted in $120 \mu \mathrm{L}$ of IPG (immobilized pH gradient) rehydration buffer. Samples were actively rehydrated and put onto 7-cm pH 3-10 IPG strips (Amersham Biosciences) using Protean IEF cell (Bio-Rad) isoelectric focusing and then separated using SDS-PAGE. 2-DE was performed in triplicate for crude antigen of EgCF under the same conditions for IgE immunoblotting, IgG1 immunoblotting, and Coomassie blue G-250 staining. Sera of T1 patient, in whom slgE and slgG1 were all positive and typical anaphylactic shock occurred due to the rupture of parasitic cyst during the surgery, was used. The method of 2-DE lgE immunoblotting experiment was the same as one-dimensional sodium dodecyl sulfatepolyacrylamide gel electrophoresis (1D SDS-PAGE) IgE immunoblotting.

IgG1 immunoblotting. Patient sera (1:20 dilution) was added with HRP-labeled mouse anti-human IgG1 antibody (1:3000 dilution). Blots were developed using SuperSignal WestPico (Thermo, Lot no: NCl508), and luminescence was detected on an X-ray film. For a clearer separation of crude EgCF antigen on the $24-\mathrm{cm}$ strip, 2-DE electrophoresis experiments were performed again, similar to the steps for the $7-\mathrm{cm} 2-\mathrm{DE}$. For the 
preparation of crude antigen of EgCF labeled with Cy3 (Cy3 DIGE kit; GE, Co., Ltd., USA), $800 \mu \mathrm{g}$ of crude antigen of EgCF was diluted in $450 \mu \mathrm{L}$ of IPG rehydration buffer. Samples were actively rehydrated into $24-\mathrm{cm}$ $\mathrm{pH}$ 3-10 IPG strips (Amersham Biosciences) using Protean IEF cell (Bio-Rad) isoelectric focusing and then separated using SDS-PAGE. Image of the EgCF separated proteins was obtained by fluorescence scanning using a laser scanner.

\section{Statistical analysis}

Statistical analysis was performed using SPSS 15.0 software (SPSS Inc., USA). Absorbance values of the healthy volunteers and patients were compared with single factor analysis of variance (ANOVA).

\section{Results}

The absorbance values in patients and disease controls are reported in Table 1 . The cutoff values of $\operatorname{lgE}$ and IgG1 were 0.64 and 2.15, respectively. Seven patients with higher $\lg E$ and eight patients with higher $\lg \mathrm{G} 1$ absorbance values than the cutoff were considered to be positive and were chosen for recognition of EgCF antigens of slgE and slgG1 using immunoblotting. In patients T1, T10, and T18, anaphylactic shock occurred during surgery and their sera were positive for slgE and slgG1.

\section{D SDS-PAGE and immunoblotting}

The protein component in the crude antigen of EgCF was analyzed using 1D SDS-PAGE. Several protein bands

Table 1. Demographic, clinical, and antibody characteristics of patients and disease controls.

\begin{tabular}{|c|c|c|c|c|c|c|c|}
\hline Patient & Gender & Age (years) & Location of cysts & Type of cysts & Anaphylactic shock & slgE Abs value & slgG1 Abs value \\
\hline T1 & $M$ & 35 & Liver & Type II & Yes & 0.92 & 2.64 \\
\hline T2 & $\mathrm{F}$ & 36 & Liver, spleen & Type II & No & 0.50 & 1.95 \\
\hline T3 & M & 36 & Liver & Type II & No & 0.49 & 1.30 \\
\hline T4 & $M$ & 33 & Liver & Type I & No & 0.62 & 1.57 \\
\hline T5 & $M$ & 42 & Liver & Type II & No & 0.64 & 2.57 \\
\hline T6 & $\mathrm{F}$ & 40 & Liver & Type II & No & 0.57 & 2.11 \\
\hline $\mathrm{T} 7$ & $M$ & 25 & Liver & Type II & No & 1.22 & 1.90 \\
\hline T8 & $M$ & 7 & Liver & Type II & No & 1.58 & 1.61 \\
\hline T9 & $\mathrm{F}$ & 16 & Liver & Type II & No & 0.46 & 2.64 \\
\hline T10 & $M$ & 42 & Liver, lung, pelvic cavity & Type II, Pulmonary & Yes & 0.94 & 2.48 \\
\hline T11 & $\mathrm{F}$ & 51 & Liver & Type II & No & 0.19 & 1.45 \\
\hline T12 & $M$ & 33 & Liver & Type II & No & 0.33 & 2.11 \\
\hline T13 & M & 21 & Liver & Type I & No & 1.01 & 2.12 \\
\hline T14 & $\mathrm{F}$ & 60 & Liver & Type II & No & 0.42 & 1.09 \\
\hline T15 & $\mathrm{F}$ & 41 & Liver & Type II & No & 0.93 & 2.40 \\
\hline T16 & $\mathrm{F}$ & 60 & Liver, pelvic cavity & Type II & No & 0.61 & 2.43 \\
\hline T17 & $M$ & 31 & Liver & Type II & No & 0.53 & 2.55 \\
\hline T18 & $\mathrm{F}$ & 10 & Lung & Pulmonary & Yes & 1.66 & 2.35 \\
\hline T19 & $\mathrm{F}$ & 46 & Liver & Type II & No & 0.43 & 2.13 \\
\hline T20 & $\mathrm{F}$ & 39 & Lung & Pulmonary & No & 0.63 & 1.36 \\
\hline C1 & $M$ & 27 & & & & 0.41 & 1.15 \\
\hline $\mathrm{C} 2$ & $\mathrm{~F}$ & 34 & & & & 0.52 & 1.53 \\
\hline C3 & $M$ & 28 & & & & 0.43 & 0.64 \\
\hline $\mathrm{C} 4$ & $\mathrm{~F}$ & 30 & & & & 0.36 & 0.41 \\
\hline C5 & $M$ & 41 & & & & 0.35 & 1.08 \\
\hline C6 & $\mathrm{F}$ & 26 & & & & 0.42 & 0.90 \\
\hline $\mathrm{C7}$ & $M$ & 28 & & & & 0.44 & 0.22 \\
\hline $\mathrm{C} 8$ & $\mathrm{~F}$ & 32 & & & & 0.55 & 0.20 \\
\hline $\mathrm{Cg}$ & $M$ & 35 & & & & 0.42 & 0.41 \\
\hline C10 & $\mathrm{F}$ & 28 & & & & 0.30 & 1.16 \\
\hline
\end{tabular}

T1-T20: patient group; C1-C10: disease control group; M: male; F: female; Yes: anaphylactic shock occurred during surgery; No: anaphylactic shock did not occur during surgery. Abs: absorbance; slgE: specific immunoglobulin E; slgG1: specific immunoglobulin G1. Cystic echinococcosis (CE) in hepatic tissues was classified according to ultrasonographic classification. Type I: simple CE: a) overall echo-free; b) with fine echoes. Type II: multiple CE: a) multiple contiguous cysts; b) multi-septated with rosette, honey-comb, or wheellike pattern. Pulmonary echinococcosis does not have its own classification. 


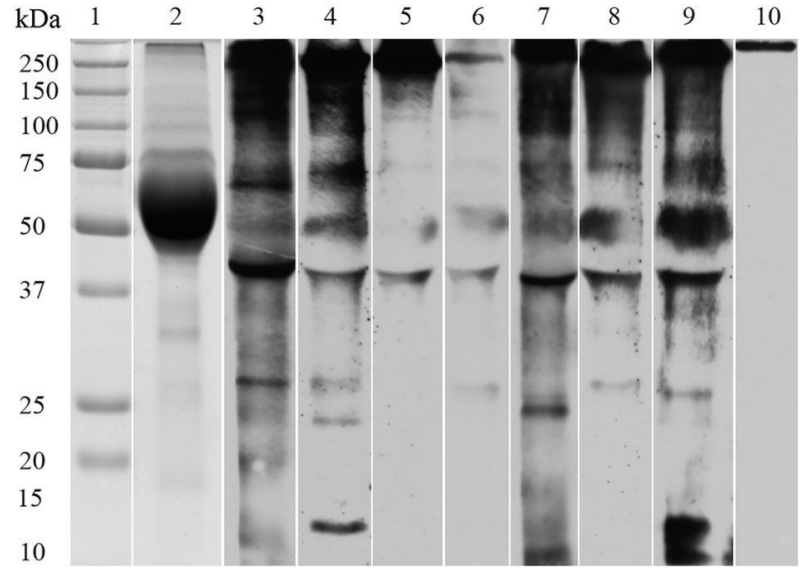

Figure 1. SDS-PAGE and $\lg E$ immunoblotting of the crude antigen of $E$. granulosus cyst fluid (EgCF). Crude antigen of EgCF protein extracts were incubated individually with 7 slgE positive sera (lanes 3-9), and with disease control (lane 10); crude antigen of EgCF proteins visualized using Coomassie blue R-250 (lane 2); protein molecular weight marker (lane 1). IgE, immunoglobulin E; SDS-PAGE, sodium dodecyl sulfate-polyacrylamide gel electrophoresis; slgE, specific immunoglobulin E.

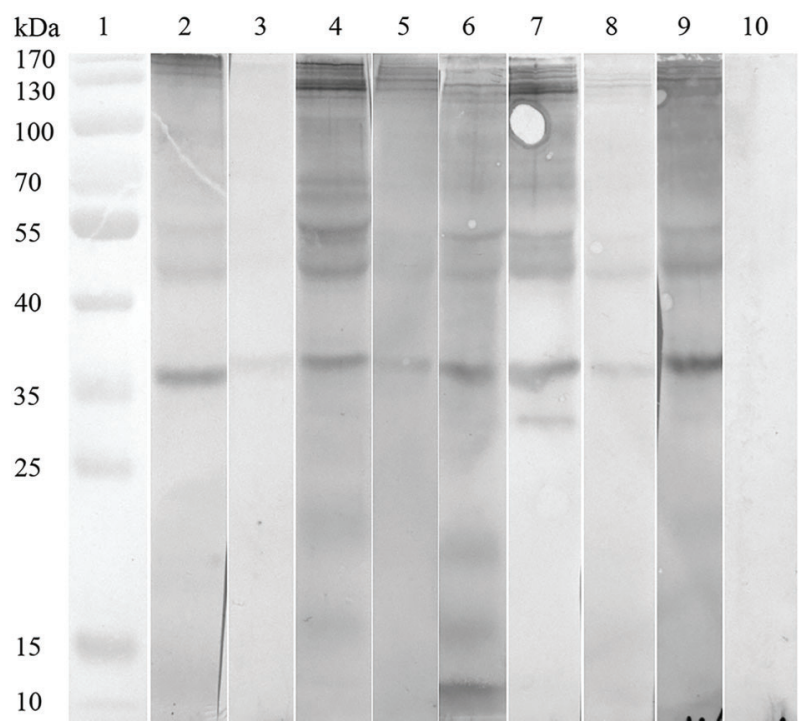

Figure 2. SDS-PAGE and $\operatorname{lgG} 1$ immunoblotting of the crude antigen of Echinococcus granulosus cyst fluid (EgCF). Crude antigens of EgCF protein extracts were incubated individually with 8 slgG1 positive sera (lanes 2-9), and with disease control (lane 10); protein molecular weight marker (lane 1). SDS-PAGE, sodium dodecyl sulfate-polyacrylamide gel electrophoresis; slgG1, specific immunoglobulin G1.

ranging from 10 to $250 \mathrm{kDa}$ were detected. The most abundant protein band had a molecular weight of 53.5 $\mathrm{kDa}$. The differences in intensity in protein molecular weight of $51.3,40.5,27.0$, and $24.5 \mathrm{kDa}$ of $\mathrm{IgE}$ reaction were detected among individual sera. The $40.5-\mathrm{kDa}$

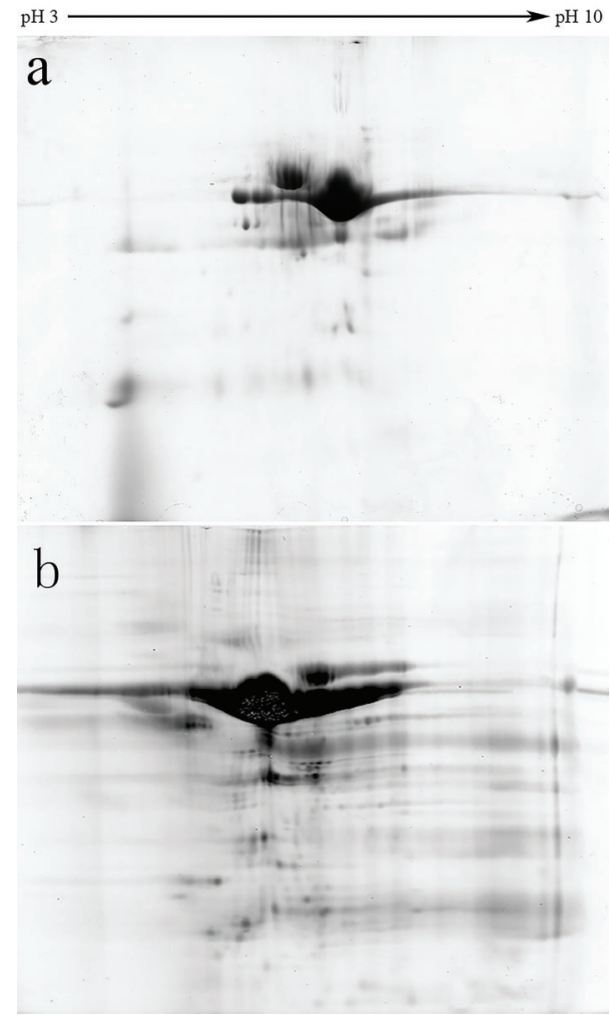

Figure 3. a, Seven-centimeter Coomassie blue-stained 2-DE gel of crude antigen of EgCF; $b$, Twenty-four-centimeters fluorescence scanning 2-DE gel of crude antigen of EgCF. 2-DE, twodimensional gel electrophoresis; EgCF, Echinococcus granulosus cyst fluid.

protein showed positive reaction with 7 slgE positive sera, and was the major antigen of slgE in EgCF. The differences in intensity in protein molecular weight of 35.5 , 44.6 , and $53.2 \mathrm{kDa}$ of $\mathrm{lgG} 1$ reaction were detected among individual sera. The $35.5-\mathrm{kDa}$ protein showed positive reaction with 8 slgG1 positive sera, and was the major antigen of slgG1 in EgCF. Sera obtained from disease controls showed no reaction against any of the EgCF proteins (Figures 1 and 2).

A representative 7-cm 2-DE gel figure of crude antigen of EgCF stained with Coomassie blue shows the visualization of about 50 protein spots ranging in molecular mass from 10 to $250 \mathrm{kDa}$, with pl values ranging from 3 to 10 . A representative 24-cm 2-DE gel figure of crude antigen of EgCF using laser scanner shows the visualization of about 200 protein spots ranging in molecular mass from 10 to $250 \mathrm{kDa}$, with pl values ranging from 3 to 10 (Figure 3 ). To identify the separated proteins, proteins of crude antigen of EgCF were transferred to a nitrocellulose membrane and incubated with $\mathrm{T} 1$ patient sera. PI values of four spots ranging from 6.5 to 9.0 comprising $40.5 \mathrm{kDa}$ protein in slgE immunoblotting and $\mathrm{pl}$ values of five spots ranging from 


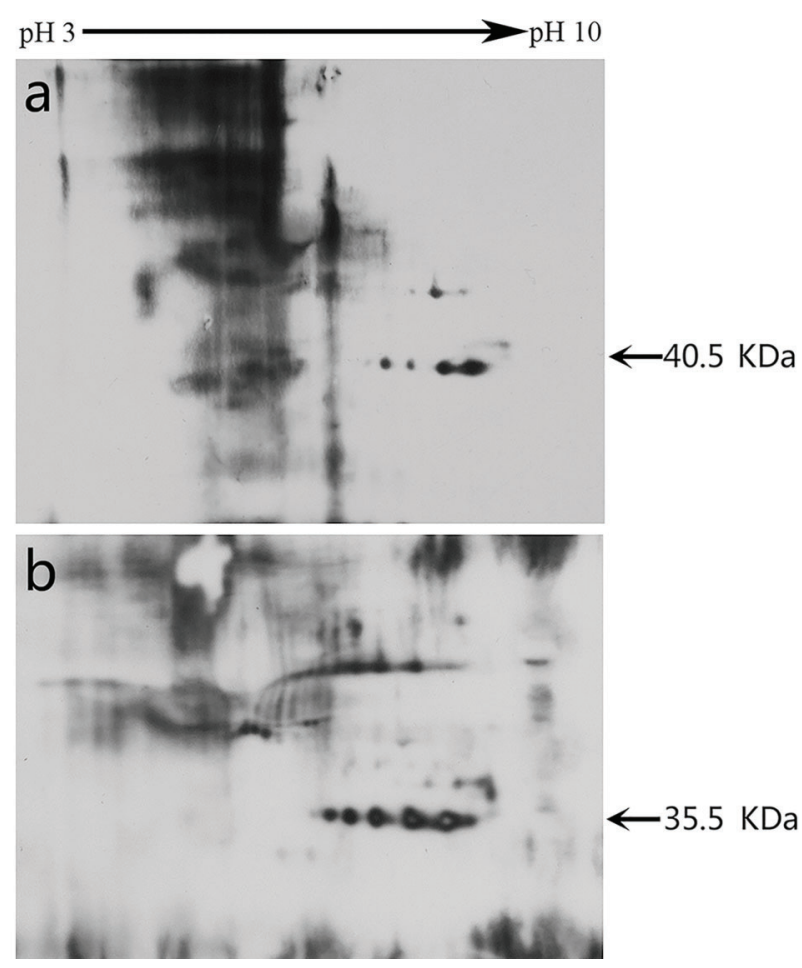

Figure 4. The crude antigen of Echinococcus granulosus cyst fluid (EgCF) proteins separated by 2-DE was transferred to the nitrocellulose membrane and probed with sera of patient T1. a, $\mathrm{lgE}$ immunoblotting was visualized using HRP-labeled mouse anti-human IgE; $b$, IgG1 immunoblotting was visualized using HRP-labeled mouse anti-human IgG1. The sizes of the prominent proteins in $\mathrm{kDa}$ are shown on the right-hand side of the figure. 2-DE, two-dimensional gel electrophoresis; IgE, immunoglobulin $\mathrm{E}$; IgG1, immunoglobulin G1.

6.5 to 9.0 comprising $35.5 \mathrm{kDa}$ protein in IgG1 immunoblotting showed strong reaction with T1 patient sera (Figure 4).

\section{Discussion}

Anaphylactic shock induced by $C E$ is mediated through slgE and slgG1 subclass. In this study, sera from 3 patients with CE who had anaphylactic shock were collected and slgE- and slgG1-positive serum were screened using SDS-PAGE, 2-DE, and immunoblotting technique for the corresponding antigen in EgCF. The main antigens of slgE in EgCF were identified with four protein spots with pl values ranging from 6.5 to 9.0 and a molecular weight of $40.5 \mathrm{kDa}$. The main antigens of slgG1 in EgCF were identified with five protein spots with pl values ranging from 6.5 to 9.0 and a molecular weight of $35.5 \mathrm{kDa}$.

In this study, indirect ELISA method was used to screen slgE- and slgG1-positive serum in 20 patients. The absorbance values in controls showed no significant differences than in patients. As plates coated with crude antigen of EgCF were derived from patients with echinococcosis containing anthropogenic $\lg \mathrm{E}$ and $\lg \mathrm{G} 1$, it may have produced false-positive results. Hence in the former experiment, EgCF from sheep liver was used to prepare the crude antigen. Seven cases of slgE-positive serum and 8 cases of slgG1-positive serum were screened using ELISA. It is interesting to note that 3 cases with anaphylactic shock were caused by spillover of EgCF during the surgery; slgE and slgG1 were all positive. The results suggest that the content of serum slgE and slgG1 is not only associated with patients with hydatid infections, but also associated with normal allergic components of patients. Results of a preliminary study showed that anaphylactic shock of patients was related to higher content of serum total $\lg E$ and $\lg G 1$ (11).

$\mathrm{lgE}$ immunoblotting results showed that the $40.5-\mathrm{kDa}$ proteins of crude antigen of EgCF had prominent reaction with all seven slgE-positive sera. In preliminary 1D SDSPAGE and IgE immunoblotting experiments using DAB, TMB (tetramethylbenzidine), and SuperSignal WestPico did not show obvious immunoblotting bands. However, the use of SuperSignal West Femto Maximum Sensitivity Substrate showed prominent reaction in IgE immunoblotting bands. This suggests that the concentration of slgE in the serum and slgE antigen in EgCF was very low. Results of slgG1 immunoblotting bands also did not distinctly show the corresponding bands or protein point on 1D SDS-PAGE and $2-D E$. The results of the study showed that the slgE and sigG1 antigen in EgCF belongs to the low abundance proteins. Removing high abundance proteins extraneous to the antigen of cystic fluid protein will concentrate low abundance proteins containing purpose antigen and effectively isolate slgE and slgG1 of EgCF antigen.

EgCF antigen for the sensitivity and specificity of specific antibodies was the core index for screening diagnostic antigen. Anaphylactic shock mediated through antibody levels is closely related to the allergic constitution and immune status of the patient; therefore, allergens may not have high sensitivity and specificity of EgCF diagnostic antigens. Studies on slgE antigen in EgCF are few, with different methods and results $(13,14)$, and the slgG1 subclass antigen in EgCF has not been reported. AgB is a polymeric lipoprotein with a molecular mass of $120-160 \mathrm{kDa}$, which under reducing electrophoretic conditions appears to be composed of three subunits with molecular sizes of 8,16 , and $24 \mathrm{kDa}$. AgB is considered to have high sensitivity and specificity in the diagnosis of echinococcosis $(15,16)$, but it cannot be identified by the slgE (10). Ag5 is a high molecular mass lipoprotein complex of 67 and $57 \mathrm{kDa}$ antigen in EgCF, which under reducing conditions dissociates into 38 and $22 \mathrm{kDa}$ subunits (13). Results of slgE and slgG1 immunoblotting showed no obvious reaction strip in the $\mathrm{AgB}$ and $\mathrm{Ag} 5$, due to their monomer molecular weight.

\section{Study limitation and future experimental plans}

In this study, valuable serum was collected only from 3 patients with echinococcosis who experienced 
anaphylactic shock. Whether the recognized antigen could identify the allergen responsible for anaphylactic shock has not yet been validated. Removing high abundance proteins extraneous to the antigen of cystic fluid protein will concentrate low abundance proteins containing target antigen, and effectively isolate slgE and slgG1 EgCF antigen. The purified and detected amino acid sequence was effective for the candidate antigens.

\section{References}

1. Budke $\mathrm{CM}$, Carabin $\mathrm{H}$, Ndimubanzi $\mathrm{PC}$, Nguyen $\mathrm{H}$, Rainwater $\mathrm{E}$, Dickey $\mathrm{M}$, et al. A systematic review of the literature on cystic echinococcosis frequency worldwide and its associated clinical manifestations. Am J Trop Med Hyg 2013; 88: 1011-1027, doi: 10.4269/ajtmh.12-0692.

2. Wang G, Feng $X$, Chu X, ErxidingAmina, Wang $Q$, et al. Epidemiological study on human echinococcosis in Hobukesar Mongolian Autonomous County of Xinjiang. Chin $J$ Endemiol 2009; 28: 214-217.

3. Saenz de San Pedro B, Cazana JL, Cobo J, Serrano CL, Quiralte J, Contreras J, et al. Anaphylactic shock by rupture of hydatid hepatic cyst. Follow-up by specific IgE serum antibodies. Allergy 1992; 47: 568-570, doi: 10.1111/j.13989995.1992.tb00683.x.

4. Qi F, Xu H, Wang Y, Dou H, Wu Y, Chen K. Experience on the rescuing anaphylactic shock caused by effusion of kidney hydatid fluid. Int J Med Parasit Dis 2009; 36: 85-86.

5. Vuitton DA. Echinococcosis and allergy. Clin Rev Allergy Immunol 2004; 26: 93-104, doi: 10.1007/s12016-0040004-2.

6. Li Y, Zheng H, Cao X, Liu Z, Chen L. Demographic and clinical characteristics of patients with anaphylactic shock after surgery for cystic echinococcosis. Am J Trop Med Hyg 2011; 85: 452-455, doi: 10.4269/ajtmh.2011.10-0448.

7. Dong N, Chen L, Xu M, Deng H, Su C, Qin C, et al. Isolation and purification of anaphylactic antibody from guinea pig and preliminary research on its role in passive cutaneous anaphylaxis test(PCA). Chin J Microbiol Immunol 2010; 30: 169-173.

8. Oettgen HC, Martin TR, Wynshaw-Boris A, Deng C, Drazen $\mathrm{JM}$, Leder P. Active anaphylaxis in IgE-deficient mice. Nature 1994; 37: 367-370, doi: 10.1038/370367a0.

9. Tsujimura $Y$, Obata K, Mukai K, Shindou H, Yoshida M, Nishikado $H$, et al. Basophils play a pivotal role in
In conclusion, slgE and slgG1 antigens in EgCF were identified, providing critical insights into cellular and molecular mechanisms underlying anaphylactic shock induced by CE.

\section{Acknowledgments}

This study was supported by the National Natural Science Foundation of China (No. 81460309).

immunoglobulin-G-mediated but not immunoglobulin-Emediated systemic anaphylaxis. Immunity 2008; 28: 581-589, doi: 10.1016/j.immuni.2008.02.008.

10. Zheng $H, X u Z X$, Yang GX, Wen $H$. [Study on the level of specific $\lg G, \lg G 1$ and $\lg E$ during anaphylactic shock in sheep induced by Echinococcus granulosus]. Zhongguo Ji Sheng Chong Xue Yu Ji Sheng Chong Bing Za Zhi 2003; 21: 42-45.

11. Li Y, Zheng $\mathrm{H}, \mathrm{Gu} M$, Cao X, Wen H, Liu Z, et al. Comparisons of serum total $\lg E, \lg G$, and $\lg \mathrm{G} 1$ levels in patients with and without echinococcosis-induced anaphylactic shock. Am J Trop Med Hyg 2012; 87: 104-108, doi: 10.4269/ajtmh.2012.11-0694.

12. Liu R, Krishnan HB, Xue W, Liu C. Characterization of allergens isolated from the freshwater fish blunt snout bream (Megalobrama amblycephala). J Agric Food Chem 2011; 59: 458-463, doi: 10.1021/jf103942p.

13. Khabiri AR, Bagheri F, Assmar M, Siavashi MR. Analysis of specific IgE and IgG subclass antibodies for diagnosis of Echinococcus granulosus. Parasite Immunol 2006; 28: 357-362, doi: 10.1111/j.1365-3024.2006.00837.x.

14. Ortona E, Vaccari S, Margutti $P$, Delunardo F, Rigano R, Profumo E, et al. Immunological characterization of Echinococcus granulosus cyclophilin, an allergen reactive with IgE and IgG4 from patients with cystic echinococcosis. Clin Exp Immunol 2002; 128: 124-130, doi: 10.1046/j.1365-2249. 2002.01807.x

15. Gao C, Wang J, Yang Y, Shi F, Zhu H, Jiao W. Serological evaluation of five Echinococcus granulosus antigens for detecting cystic echinococcosis by ELISA. Chin J Zoonoses 2012; 28: 811-814.

16. Li $Y$, Meng $C$, Zhang Z. Diagnostic value of ELISA using antigen $B$ in cystic echinococcosis: A meta-analysis. Chin $J$ Evidence-Based Med 2013; 13: 332-338. 